\title{
Random mutagenesis by insertion of error-prone PCR products to the chromosome of Bacillus subtilis
}

\author{
Be $\mathrm{Ye}^{1}, \mathrm{Yu} \mathrm{Li}^{1}$, Qin $\mathrm{Tao}^{1}$, Xiaoliang $\mathrm{Yao}^{1}$, Minggen Cheng${ }^{1}$, and Xin $\mathrm{Yan}^{1}$ \\ ${ }^{1}$ Nanjing Agricultural University
}

May 15, 2020

\begin{abstract}
Bacillus subtilis is an attractive host for directed evolution of the enzymes whose substrates cannot be transported across the cell membrane. However, generation of mutant library in B. subtilis still suffers problems of small library size, plasmid instability and heterozygosity. Here, large library of random mutant was created through inserting error-prone PCR (epPCR) product to the chromosome of B. subtilis. Specifically, epPCR product was fused with flanking regions and antibiotic resistant marker using a PCR-based multimerization method, generating insertion construct. epPCR product was integrated into chromosome via homologous recombination after insertion construct was transformed into the supercompetent cells of B. subtilis strain SCK6. The transformation efficiency of insertion construct was improved though increasing the number of competent cell and the length of flanking regions. A library containing $3.5 \times 105$ random mutant was construction using per $\mu$ insertion construct, which is sufficient for directed evolution. Moreover, the library generation process could be accomplished within one day. The effectiveness of this method was confirmed by improving the activity of Methyl Parathion Hydrolase (MPH) toward chlorpyrifos and to enhance the secretion level of MPH in B. subtilis. Taken together, present work provides a fast and efficient method to integrate epPCR product into the chromosome of B. subtilis, facilitating directed evolution and expression optimization of target protein.
\end{abstract}

\section{Keywords}

epPCR, Chromosomal integration, Large library, Directed evolution,

Bacillus subtilis

\section{INTRODUCTION}

Directed evolution has proved to be a powerful tool to improve the activity, stability and substrate specificity of enzymes (Goldsmith et al. 2017; Romero et al. 2009; Roodveldt et al. 2005; Wong et al. 2004; Wong et al. 2007; Zeymer et al. 2018). This tool involves two crucial steps: generation of a library containing sufficient gene variants and high-throughput screening of library members with desired properties (Packer et al. 2015; Wong et al. 2006). Because of its high transformation efficiencies, rapid growth rates, wellestablished manipulation approaches (Crameri et al. 1996), Escherichia coliis generally employed as host organism for library creation. However, proteins are usually expressed in cytoplasm in E. coli, making the screening of library more difficult if the substrate of the protein cannot be transported into cell. Therefore, the organisms such asBacillus subtilis and Pichia pastoris that can secret proteins into medium have been developed as alternative hosts for library generation (Jiang et al. 2019; Liu et al. 2017; Reetz et al. 2007; Wang et al. 2012).

B. subtilis is an important industrial host for production of various recombinant proteins due to its GRAS (generally recognized as safe) status, excellent protein secretion ability and mature fermentation processes (Commichau et al. 2014; Marcus et al. 2004; Schumann et al. 2007; Terpe et al. 2006; Yang et al. 
2011). Although B. subtilis has well-developed genetic manipulation tools, its transformation frequency is still much lower than that of $E$. coli . Thus, the library of random mutants generated through digestion and ligation is too small to fulfill the need of direct evolution in $B$. subtilis . Several strategies have been adopted to address this limitation. A routine strategy is first constructing the library of variants in $E$. coli and then transferring the library into $B$. subtilis, which is time-consuming and of low efficiency (Caspers et al. 2010; You et al. 1996; Zhao et al. 1999). Melnikov et al. (1999) attempted to clone error-prone PCR (epPCR) product via marker-replacement recombination with a structurally similar helper plasmid resident in the transformation recipient. But they found that it was difficult to recover $>10^{3}$ transformants $/ \mu \mathrm{g}$ of epPCR product. Given the phenomenon that multimeric plasmid has much higher (approximately three orders of magnitude) transformation frequency than that of monomeric plasmid in B. subtilis (Canosi et al. 1978), Shafikhani et al. (1997) generated large libraries of random mutants $\left(3 \times 10^{6}\right)$ in B. subtilis by PCR-based plasmid multimerization method. In this method, epPCR product was fused with linearized vector through PCR extension to generate linear plasmid multimer which could be converted to circular form through homologous recombination after entering cell; however, each cell may take several variants, decreasing screening efficiency. Increasing the number of competent cells is an effective method to create larger library. To this end, Zhang and Zhang et al. (2011) constructed a B. subtilis strain SCK6 whose competence can be induced by artificially controlling the expression of master regulator ComK. The procedure for preparing supercompetent cell of strain SCK6 was further improved by Li et al. (2017). The transformation frequencies of multimeric plasmid, monomeric plasmid and integration plasmid into strain SCK6 could reach $10^{7}, 10^{4}$ and $10^{5}$ transformants/ $\mu \mathrm{g}$ DNA.

This study aims to construct large mutant library through inserting epPCR product into the chromosome of $B$. subtilis, which will solve the problems of plasmid instability and heterozygosity faced by multicopy plasmid mediated strategies (Melnikov et al. 1999; You et al. 2012; Zhang and Zhang 2011). We accomplished the library construction within one day and the generated library had sufficient mutants $\left(>10^{5}\right)$ for the need of direct evolution.

\section{MATERIALS AND METHODS}

\subsection{Bacterial strains, primers and growth conditions}

The B. subtilis SCK6 strain was provided by Daniel R. Zeigler from the Bacillus Genetic Stock Center. Oligonucleotide synthesis (Table 1) and DNA sequencing were performed by Sangon Biotech Co., Ltd. (Shanghai, China). B. subtilis strains were cultivated in Lysogeny Broth (LB) (Bertani et al. 2004) medium, YN medium (Li et al. 2017) or $2 \times$ Super-Rich $(2 \times$ SR) (Song et al. 2017) medium. LB medium consists of $1 \%$ tryptone, $0.5 \%$ yeast extract and $0.5 \% \mathrm{NaCl}$. YN medium is composed of $0.7 \%$ yeast extract and $1.8 \%$ nutrient broth. $2 \times \mathrm{SR}$ medium (3\% tryptone, $5 \%$ yeast extract and $0.6 \% \mathrm{~K}_{2} \mathrm{HPO}_{4}, \mathrm{pH} 7.2$ ) was used for fermentation. Solid medium was obtained by adding $15 \mathrm{~g} / \mathrm{L}$ agar to the liquid medium. Unless otherwise indicated, the final concentrations of antibiotics were as follows $(\mathrm{mg} / \mathrm{L})$ : zeocin (Zeo), 20; erythromycin (Em), 5. The inoculums (1\%) were transferred into $250 \mathrm{~mL}$ flasks containing $30 \mathrm{~mL} 2 \times \mathrm{SR}$ medium, and incubated at 37 with shaking at $200 \mathrm{rpm}$. Chlorpyrifos (>99\%) was purchased from the Macklin Biochemical Technology Co., Ltd. (Shanghai, China).

\subsection{Construction of Methyl Parathion Hydrolase (MPH) secretion strain}

Four DNA fragments of $P_{\text {cry3A }}$ promoter (Adams et al. 1994), the coding region of signal peptide ( $\mathrm{SP}_{\text {aprE }}$ ), MPH-encoding gene mpd (Zhang et al. 2005), and T1T2 transcription terminator (Hartl et al. 2001) were fused by overlap PCR (Shevchuk et al. 2004) using the primers listed in Table 1, generating MPH expression cassette $P_{\text {crysA }}$ - mpd. The expression cassette was then fused with flanking regions of amyE and Zeo resistant marker $\left(\mathrm{Zeo}^{\mathrm{R}}\right)$, producing insertion construct. Insertion construct was transformed into the competent cells of strain SCK6 and selected by Zeo.P $P_{\text {crysA }}-m p d$ was finally integrated at the locus of amyE of strain SCK6, resulting in strain BPC1.

\subsection{Generation of MPH mutant library}


The right flanking region (RF) of amyE locus was amplified from strain BPC1 using primer pair P1/P5. Another fragment containing left flanking region $(\mathrm{LF}), \mathrm{Zeo}^{\mathrm{R}}$ and $P_{\text {crys } A}$ was amplified from strain $\mathrm{BPC} 1$ using primer pair P6/P2. These two fragments were fused by overlap PCR (Shevchuk et al. 2004) using primer pair P1/P2, resulting in fragment RF-LF-Zeo ${ }^{\mathrm{R}}-P_{\text {cry3A }}$. The cleavage site Sac I was introduced between RF and LF.

The coding region of $\mathrm{SP}_{\mathrm{aprE}}$ and MPH was amplified by error-prone PCR with primer pair P3/P4 using the Starmut Random Mutagenesis Kit (GenStar, Beijing, China). The PCR reaction solution with a total volume of $50 \mu \mathrm{L}$ contained $0.02 \mathrm{ng} / \mu \mathrm{L}$ template, $25 \mu \mathrm{L} 2 \times$ buffer, $3 \mu \mathrm{L}$ StarMut Enhancer, $0.4 \mathrm{mM}$ primer pair P3/P4. The PCR was conducted as follows: initial denaturation at $95{ }^{\circ} \mathrm{C}$ for 2 min and subsequent steps of $94{ }^{\circ} \mathrm{C}$ for $30 \mathrm{~s}$, annealing at $56{ }^{\circ} \mathrm{C}$ for $1 \mathrm{~min}$, and extension at $72{ }^{\circ} \mathrm{C}$ for $1 \mathrm{~min}$ for 30 cycles total. The overlap of $47 \mathrm{bp}$ between the left end of the epPCR product and the right end of RF-LF-Zeo ${ }^{\mathrm{R}}-P_{\text {cry }} 3 \mathrm{~A}$ was introduced through primers $\mathrm{P} 4$ and P1, and the overlap of $45 \mathrm{bp}$ between the right end of the epPCR product and the left end of RF-LF-Zeo ${ }^{\mathrm{R}}-P_{\text {crysA }}$ was introduced through primers P3 and P2.

To generate the multimer of fragment $\mathrm{LF}-\mathrm{Zeo}^{\mathrm{R}}-P_{\text {crys }}-m p d-\mathrm{RF}$, each $50 \mu \mathrm{L}$ reaction system contained 0.2 $\mathrm{mM}$ dNTP, equal molar amount of RF-LF-Zeo ${ }^{\mathrm{R}}-P_{\text {crys }}$ a and epPCR product ( $\sim 30$ pmol for each fragment), and $0.04 \mathrm{U} / \mu \mathrm{L}$ Phusion polymerase without the addition of primers. The prolonged overlap extension PCR was conducted as follows: denaturation at $98{ }^{\circ} \mathrm{C}$ for $30 \mathrm{~s} ; 30$ cycles of denaturation at $98{ }^{\circ} \mathrm{C}$ for $15 \mathrm{~s}$, annealing at $60{ }^{\circ} \mathrm{C}$ for $15 \mathrm{~s}$, and extension at $72{ }^{\circ} \mathrm{C}$ at a rate of $2 \mathrm{~kb} / \mathrm{min}$; followed by $72{ }^{\circ} \mathrm{C}$ extension for $10 \mathrm{~min}$. The multimer product was digested with Sac I to release the monomer of fragment LF-Zeo ${ }^{\mathrm{R}}-P_{c r y 3 A}-m p d-\mathrm{RF}$ which was transformed into the competent cells of strain SCK6 according to following procedure.

\subsection{Transformation of $B$. subtilis}

The B. subtilis SCK6 strain was inoculated into $4 \mathrm{ml}$ of YN medium with $5 \mathrm{mg} / \mathrm{L}$ erythromycin in a test tube. The cells were cultivated at $37^{\circ} \mathrm{C}$ with shaking at $220 \mathrm{rpm}$ overnight $(\sim 12 \mathrm{~h})$. The culture was diluted to an optical density at $600 \mathrm{~nm}$ (OD600) of 1.0 in a fresh YN medium containing $1.5 \%$ (w/v) xylose and then was grown for $2 \mathrm{~h}$. The resulting cell culture was ready to be transformed. $100 \mathrm{ng}$ of DNA was mixed with different volumes of the supercompetent cells in a 1.5-ml eppendorf tube and cultivated at $37{ }^{\circ} \mathrm{C}$ with shaking at $220 \mathrm{rpm}$ for $90 \mathrm{~min}$.

\subsection{Screening of the mutant library}

Transformants were selected on LB plates containing Zeo and colonies were then transferred to LB plates containing $50 \mathrm{mg} / \mathrm{L}$ chlorpyrifos using sterile toothpick. Strain BPC1was used as control. After incubation at 37 for $12 \mathrm{~h}$, the MPH activity of each colony was determined by the size of transparent halos. Mutants with larger halo were selected for further verification.

\subsection{MPH purification and chlorpyrifos-hydrolyzing}

\section{activity assay}

MPH purification was performed following the method described by Zhao et al. (2019). Briefly, the cells harboring MPH expression cassette were grown in $2 x S R$ medium for $36 \mathrm{~h}$ at 37 . The supernatant of the cultures was used to purify C-terminal His-tagged MPH through Ni-NTA affinity chromatography according to the manufacturer's instructions (Sangon Biotech Co., Ltd., Shanghai, China). Protein concentration was measured by the BCA Kit (Shanghai, Sangon Biotech Co., Ltd., China). Target proteins were analyzed by SDS-PAGE and the gel was stained with Coomassie brilliant blue R250. The activity of MPH toward chlorpyrifos was measured as described previously (Xie et al. 2014).

\subsection{Site-directed mutagenesis}

Each mutation was introduced into wildtype $P_{c r y 3 A}-m p d$ expression cassette through overlap PCR (Shevchuk et al. 2004). The genomic DNA of strain BPC1 was used as template and the primers were listed in Table 2. The PCR product was directly transformed into strain SCK6, generating the desired mutants. 


\section{RESULTS}

\subsection{Scheme for random mutagenesis by insertion of PCR products to the chromosome}

As shown in Figure 1, error-prone PCR (epPCR) product is firstly assembled into an insertion construct consisting of left flanking region (LF), antibiotic resistant marker $\left(A b^{R}\right)$, epPCR product and right flanking region (RF); then, after the insertion construct is transformed into B. subtilis competent cells, epPCR product is inserted into the chromosome of $B$. subtilis through homologous recombination. Efficient assembly of insertion construct is the crucial step of this method. The plasmid multimerization method (Shafikhani et al. 1997; You et al. 2012) was employed to assemble insertion construct. First, LF, RF and $\mathrm{Ab}^{\mathrm{R}}$ are fused together through overlap PCR (Shevchuk et al. 2004) generating fragment RF-LF-Ab ${ }^{\mathrm{R}}$; notably, RF is put ahead of LF and a cleavage site of restriction enzyme is introduced between RF and LF. Gene of interest (GOI) is amplified by epPCR. The left end of GOI overlaps (40-50 bp) with the right end of RF$\mathrm{LF}-\mathrm{Ab}^{\mathrm{R}}$, while the right end of the GOI overlaps (40-50 bp) with the left end of RF-LF-Ab ${ }^{\mathrm{R}}$. Then, equal molar amount of GOI and RF-LF-Ab ${ }^{\mathrm{R}}$ are mixed, the multimer of insertion construct (LF-Ab ${ }^{\mathrm{R}}-\mathrm{GOI}-\mathrm{RF}$ ) is generated by prolonged overlap extension PCR. Finally, the multimer is cut at the cleavage site between RF and LF, releasing the monomer of insertion construct.

\subsection{Development of the random mutagenesis system}

The above scheme was tested by direct evolution of Methyl Parathion Hydrolase (MPH) to improve its activity toward chlorpyrifos, a pesticide that often contaminates food and environment (Lu et al. 2013). To begin with, secretion expression of MPH was realized through inserting MPH expression cassette at the amyE locus of $B$. subtilis ; the transcription of $m p d$ is driven by promoter $P_{\text {crysA }}$ and the secretion of MPH is mediated by signal-peptide of $\mathrm{AprE}\left(\mathrm{SP}_{\mathrm{AprE}}\right)$ (Figure 2a). Then, the coding region of $\mathrm{SP}_{\mathrm{AprE}}$ and $\mathrm{MPH}$ was amplified by epPCR, and another fragment of RF-cleavage site (Sac I)-LF-Zeo ${ }^{\mathrm{R}}-P_{\text {crys }}$ w was generated via overlap PCR. After multimerization, the product was converted to monomer of insertion construct (LFZeo ${ }^{\mathrm{R}}$-Pcry3A-mpd-RF) by Sac I digestion, which was confirmed by agarose gel electrophoresis. As shown in Figure $2 \mathrm{~b}$, neither of the two fragments (RF-LF-Zeo ${ }^{\mathrm{R}}-P_{\text {cry3A }}$ and epPCR product) could be detected on gel after multimerization, indicating most of the two fragments were transformed to multimers. Sac I digestion product matched the size of insertion construct, which was further verified by DNA sequencing. To enhance the transformation efficiency of insertion construct, the amount of competent cell was increased for each transformation reaction. As shown in Figure 2c, when $400 \mu \mathrm{L}$ competent cells were mixed with $100 \mathrm{ng}$ DNA, the transformation efficiency was highest reaching $2.71 \times 10^{5} \mathrm{CFU} / \mu \mathrm{g}$ DNA. Additionally, the transformation efficiency was positive correlated with the length of flanking region. The flanking region of $0.5,1$ and $1.5-\mathrm{kb}$ at each side led to the transformation efficiency of $1.24 \times 10^{5}, 2.59 \times 10^{5}$ and $3.53 \times 10^{5} \mathrm{CFU} / \mu \mathrm{g}$ DNA (Figure $2 \mathrm{~d}$ ), respectively. The flanking region of $1 \mathrm{~kb}$ at each side of insertion construct was used to generate a library of $m p d$ variants. Ten clones were randomly selected, and DNA sequencing result of $\mathrm{SP}_{\mathrm{AprE}}$ and MPH coding region shows that each mutant harbored 1-5 mutations, with an overall mutation rate of $0.31 \%$.

\subsection{Screening of the mutant library of MPH variants}

As shown in Figure 3a, the mutants were grown on LB plate containing $50 \mathrm{mg} / \mathrm{L}$ chlorpyrifos. A transparent halo could form around the colony when chlorpyrifos is hydrolyzed by MPH (Xie et al. 2014). Enlarged halo indicated enhanced MPH activity, which may be caused by improved catalytic efficiency or increased MPH number. Two mutants, named MT1 and MT2, which formed significantly bigger transparent halos were screened from about 12000 colonies. When grown in liquid medium, the growth curves of both mutants were similar with that of strain BPC1. (data not shown), but the maximum extracellular MPH activity of mutants MT1 and MT2 were 2.47 and 2.77 fold that of strain BPC1 (Figure 3b), respectively.

\subsection{Identification of the effect of each mutation on MPH}

The $\mathrm{SP}_{\mathrm{AprE}}$ and $\mathrm{MPH}$ coding region of mutants MT1 and MT2 was sequenced. The mutation sites were shown in Figure 4a. Mutant MT1 contained three mutations, with two (T47C and G81T) located in $\mathrm{SP}_{\mathrm{AprE}}$ coding region and one (G938A) located in the MPH coding region. Among these three mutations, two led 
to amino acid change (Leu16Ser and Arg313His), while G81T (Ala27Ala) is a synonymous mutation. All three mutations of mutant MT2 located in MPH coding region (T806A, C821T and C892T), resulting in the amino acid substitutions of Ile269Ser, Ala274Val and Pro298Ser.

To determine the contribution of each mutation to the enhanced extracellular MPH activity of mutants MT1 and MT2, each mutation was individually introduced into strain BPC1, generating mutants MT-16, MT-27, MT-269, MT-274, MT-298 and MT-313. All six mutations did not affect the growth of their hosts compared with strain BPC1 (data not shown). The extracellular MPH activity of mutants MT-16, MT27, MT-269, MT-298 and MT-313 were 1.73, 1.40, 1.75, 1.48 and 2.03 fold that of strain BPC1, while mutant MT-274 showed negative effect on the extracellular MPH activity (Figure 4b). The extracellular MPH of mutants MT-16, MT-27, MT-269, MT-298 and MT-313 were purified through Ni-NTA affinity chromatography. The MPH protein yield of MT-269, MT-298 and MT-313 were close to that of strain BPC1, while the MPH protein yield of MT-16 and MT-27 were 1.70 and 1.46 fold that of strain BPC1 (Figure 4d). Moreover, the specific activity of variants T806A, C892T and G938A were 1.53, 1.43 and 1.52 fold of wild-type MPH, while the variants T47C and G81T were comparable with that of wild-type MPH (Figure 4c). Taken together, mutations T47C and G81T in the $\mathrm{SP}_{\mathrm{AprE}}$ coding region enhanced extracellular MPH activity through increasing MPH protein yield, while the contribution of mutations T806A, C892T and G938A was enhancing the catalytic activity of MPH against chlorpyrifos. Additionally, to further enhance the extracellular MPH activity, two new mutants were created through recombining the mutations. Mutant MT-C2 harbors mutations T47C and T806A, and mutant MT-C3 contains mutations of T47C, T806A and G938A. The extracellular MPH activities of mutants MT-C2 and MT-C3 were 2.78 and 3.32 fold that of strain BPC1 (Figure 4e and 4f), respectively, indicating that the contribution of these mutations could be accumulated when combined together.

\section{DISCUSSION}

In present work, to efficiently insert epPCR product into the chromosome of B. subtilis, we first employed a PCR based multimerization method to fuse epPCR product with flanking region and $\mathrm{Ab}^{\mathrm{R}}$; then, the insertion construct was transformed into supercompetent cells using a modified protocol, generating $3.53 \times 10^{5}$ transformations $/ \mu \mathrm{g}$ DNA. The library could be created within one day and the library is large enough for the need of directed evolution $\left(10^{4}-10^{5}\right.$ mutants are usually screened in directed evolution). Although the library generated here is smaller than those constructed by multimeric plasmid method (Li et al. 2017; Zhang and Sathitsuksanoh et al. 2011), our method solves the problems of plasmid instability and heterozygosity encountered in plasmid based method.

A crucial step in present method is the assembly of the insertion construct (LF-Ab ${ }^{R}$-GOI-RF). We had attempted to fuse the three fragments (LF-Ab ${ }^{\mathrm{R}}$, GOI and RF) through PCR extension and Gibson assembly, but both methods resulted in low yield of insertion construct. On contrary, PCR based multimerization method (Shafikhani et al. 1997; You et al. 2012) realized efficient assembly of insertion construct (Figure $2 \mathrm{~d}$ ). Another key step is competent cell preparation and transformation. Strain SCK6 was grown in YN medium and induced by $1.5 \%$ xylose, which remarkably improves transformation efficiency as described by $\mathrm{Li}$ et al. (2017). We further demonstrated that increasing the amount of competent cell and the length of flanking region can enhance the transformation efficiency of the insertion construct (Figure 2c).

Plasmid or chromosome is used to carry expression cassette when producing a protein in B. subtilis. Plasmid mediated protein expression has the limitations of plasmid instability and safety concern (the use of $A b^{R}$ ) (Bron et al. 1991). Chromosomal integration manner can overcome the limitations of plasmid manner, but low gene dose usually leads to low protein yield. Therefore, transcription and translation level of GOI need to be dramatically enhanced to achieve high protein yield in integration manner. Mutant MT1 harbors two mutations in $\mathrm{SP}_{\mathrm{AprE}}$ coding region, both of which could improve the yield of $\mathrm{MPH}$ (Figure $4 \mathrm{~b}$ and $4 \mathrm{~d}$ ). Nijland et al. (2007) reported that a single amino acid change remarkably increased the secretion level of $\beta$-toxin. Therefore, the random mutagenesis strategy established here can find its application in improving protein secretion level in B. subtilis . 


\section{CONFLICTS OF INTEREST}

The authors declare no conflicts of interest.

\section{AUTHOR CONTRIBUTIONS}

XY developed the project idea and revised the manuscript. BY performed most of the experiments, analyzed the data, and prepared the manuscript. YL, QT and XY did some data analysis, performed some experiments. MC provided consultation for the work and contributed significantly to the preparation of the manuscript. All authors reviewed the manuscript and agreed with the content.

\section{ACKNOWLEDGMENTS}

This work was supported by National key R\&D program of China 2019YFA0905200 and National Natural Science Foundation of China (31770125 and 31970099). The authors would like to acknowledge Dr. Daniel R. Zeigler from the Bacillus Genetic Stock Center for providing the B. subtilis SCK6 strain.

\section{REFERENCES}

Adams, L. F., Mathewes, S., O'Hara, P., Petersen, A., \& Hanne Gürtler (1994). Elucidation of the mechanism of cryIIIa overproduction in a mutagenized strain of Bacillus thuringiensis var.tenebrionis . Mol Microbiol, 4(2), 381-9. doi: 10.1111/j.1365-2958.1994.tb01298.x

Bertani, G. (2004). Lysogeny at mid-twentieth century: P1, P2, and other experimental systems. J Bacteriol , 186(3), 595-600. doi: 10.1128/JB.186.3.595-600.2004

Canosi, U., Morelli, G., \& Trautner, T. A. (1978). The relationship between molecular structure and transformation efficiency of someS. aureus plasmids isolated from B. subtilis . Mol Gen Genet, 166(3), 259-67. doi: $10.1007 / \mathrm{BF} 00267617$

Caspers, M., Brockmeier, U., Degering, C., Eggert, T., \& Freudl, R. (2010). Improvement of Sec-dependent secretion of a heterologous model protein in Bacillus subtilis by saturation mutagenesis of the $\mathrm{N}$-domain of the AmyE signal peptide. Appl Microbiol Biotechnol , 86(6), 1877-85. doi: 10.1007/s00253-009-2405-x

Commichau, F. M., Alzinger, A., Sande, R., Bretzel, W., Meyer, F. M., Chevreux, B., Wyss, M., Hohmann, H. P., \& Pragai, Z. (2014). Overexpression of a non-native deoxyxylulose-dependent vitamin B6 pathway in Bacillus subtilis for the production of pyridoxine.Metab Eng , 25, 38-49. doi: 10.1016/j.ymben.2014.06.007

Crameri, A., Whitehorn, E. A., Tate, E., \& Stemmer, W. P. C. (1996). Improved green fluorescent protein by molecular evolution using DNA shuffling. Nat Biotechnol , 14, 315-319. doi: 10.1038/nbt0396-315

Goldsmith, M., \& Tawfik, D. S. (2017). Enzyme engineering: Reaching the maximal catalytic efficiency peak. Curr. Opin. Struct Biol , 47, 140-150. doi: 10.1016/j.sbi.2017.09.002

Hartl, B., Wehrl, W., Wiegert, T., Homuth, G., \& Schumann, W. (2001). Development of a new integration site within the Bacillus subtilis chromosome and construction of compatible expression cassettes. $J$ Bacteriol , 183, 4393-4393. doi: 10.1128/JB.183.8.2696-2699.2001.

Jiang, Z., Niu, T. F., Lv, X. Q., Liu, Y. F., Li, G. H., Lu, W., Du, G. C., Chen, J., \& Liu, L. (2019). Secretory Expression Fine-Tuning and Directed Evolution of Diacetylchitobiose Deacetylase by Bacillus Subtilis . Appl Environ Microbiol , 85(17), e01076-19. doi: 10.1128/AEM.01076-19.

Li, X. Z., Lu, Z. H., Zhou, Y. L., Li, S. Y., \& Zhang, G. M. (2017). Preparation and transformation optimization for supercompetent B. subtilis SCK6 cells. Chin J Biotech , 33(4), 692-698. doi: 10.13345/j.cjb.160362. 
Li, Y. X., Yi, P., Yan, Q. J., Qin, Z., Liu, X. W., \& Jiang, Z. Q. (2017). Directed evolution of a $\beta$-mannanase fromrhizomucor mieheito improve catalytic activity in acidic and thermophilic conditions. Biotechnol Biofuels , 10, 143. doi: 10.1186/s13068-017-0833-x.

Liu, X., Liang, M., Liu, Y., \& Fan, X. (2017). Directed evolution and secretory expression of a pyrethroidhydrolyzing esterase with enhanced catalytic activity and thermostability. Microb Cell Fact , 16(1), 81. doi: 10.1186/s12934-017-0698-5.

Lu, P., Li, Q., Liu, H., Feng, Z., Yan, X., Hong, Q., \& Li, S. P. (2013). Biodegradation of chlorpyrifos and 3,5,6-trichloro-2-pyridinol by cupriavidus sp. dt-1. Bioresour Technol, 127, 337-42. doi: 10.1016/j.biortech.2012.09.116.

Marcus, S., Ajay, S., \& Ward, O. P. (2004). Developments in the use ofBacillus species for industrial production. Can JMicrobiol , 50, 1. doi: 10.1139/w03-076.

Melnikov, A., \& Youngman, P. J. (1999). Random mutagenesis by recombinatorial capture of PCR products in Bacillus subtilis andAcinetobacter calcoaceticus . Nucleic Acids Res , 27, 1056-1062. doi: 10.1093/nar/27.4.1056.

Nijland, R., Heerlien, R., Hamoen, L. W., \& Kuipers, O. P. (2007). Changing a single amino acid in Clostridium perfringens beta-toxin affects the efficiency of heterologous secretion by Bacillus subtilis . Appl Environ Microbiol , 73, 1586-1593. doi: 10.1128/AEM.02356-06.

Packer, M., \& Liu. D. (2015). Methods for the directed evolution of proteins. Nat Rev Genet, 16, 379-394. doi: $10.1038 / \operatorname{nrg} 3927$.

Reetz, M. T., Carballeira, \& José Daniel (2007). Iterative saturation mutagenesis (ISM) for rapid directed evolution of functional enzymes. Nat Protoc , 2(4), 891-903. doi: 10.1038/nprot.2007.72.

Romero, P. A., Arnold, \& F. H. (2009). Exploring protein fitness landscapes by directed evolution. Nat Rev Mol Cell Biol , 10, 866-876. doi: 10.1038/nrm2805.

Roodveldt, C., Aharoni, A., \& Tawfik, D. S. (2005). Directed evolution of proteins for heterologous expression and stability. Curr Opin Struct Bioi , 15, 50-56. doi: 10.1016/j.sbi.2005.01.001.

Schumann, W. (2007). Production of recombinant proteins inBacillus subtilis . Adv Appl Microbiol , 62, 137-189. doi: 10.1016/S0065-2164(07)62006-1.

Shafikhani, S., Siegel, R. A., Ferrari, E., \& Schellenberger, V. (1997). Generation of large libraries of random mutants inBacillus subtilis by PCR-based plasmid multimerization.Biotechniques , 23, 304-310. doi: 10.2144/97232rr01.

Shevchuk, N. A., Bryksin, A. V., Nusinovich, Y. A., Cabello, F. C., Sutherland, M., \& Ladisch, S. (2004). Construction of long DNA molecules using long PCR-based fusion of several fragments simultaneously. Nucleic Acids Res , 32, e19. doi: 10.1093/nar/gnh014.

Song, Y., Fu, G., Dong, H., Li, J., Du, Y., \& Zhang, D. (2017). High-Efficiency Secretion of beta-Mannanase in Bacillus subtilisthrough Protein Synthesis and Secretion Optimization. J Agric Food Chem , 65, 25402548. doi: 10.1021/acs.jafc.6b05528.

Terpe, K. (2006). Overview of bacterial expression systems for heterologous protein production: from molecular and biochemical fundamentals to commercial systems. Appl Microbiol Biot , 72, 211-222. doi: 10.1007/s00253-006-0465-8.

Wang, X. J., Peng, Y. J., Zhang, L. Q., Li, A. N., \& Li, C. (2012). Directed evolution and structural prediction of cellobiohydrolase II from the thermophilic fungus chaetomium thermophilum. Appl Microbiol Biotechnol , 95(6), 1469-1478. doi: 10.1007/s00253-011-3799-9. 
Wong, T. S., Roccatano, D., \& Schwaneberg, U. (2007). Steering directed protein evolution: strategies to manage combinatorial complexity of mutant libraries. Environ Microbiol , 9(11), 2645-2659. doi: 10.1111/j.1462-2920.2007.01411.x.

Wong, T. S., Tee, K. L., Hauer, B., \& Schwaneberg, U. (2004) Sequence saturation mutagenesis (SeSaM): a novel method for directed evolution.Nucleic. Acids. Res. 32, e26. doi: 10.1093/nar/gnh028.

Wong, T. S., Roccatano, D., Zacharias, M., \& Schwaneberg, U. (2006). A statistical analysis of random mutagenesis methods used for directed protein evolution. J. Mol. Biol . 355(4), 0-871. doi: 10.1016/j.jmb.2005.10.082.

Xie, J., Zhao, Y., Zhang, H., Liu, Z., \& Lu, Z. (2014). Improving methyl parathion hydrolase to enhance its chlorpyrifos-hydrolysing efficiency. Lett. Appl. Microbiol . 58(1), 53-59. doi: 10.1111/lam.12155.

Yang, H., Liu, L., Li, J., Du, G., \& Chen, J. (2011). Heterologous expression, biochemical characterization, and overproduction of alkaline alpha-amylase from Bacillus alcalophilus in Bacillus subtilis . Microb. Cell. Fact. 10, 77. doi: 10.1186/1475-2859-10-77.

You, C., Zhang, X. Z., \& Zhang, Y. H. P. (2012). Simple cloning via direct transformation of pcr product (DNA multimer) to Escherichia coli and Bacillus subtilis . Appl Environ Microbiol , 78(5), 1593-1595. doi: 10.1128/AEM.07105-11.

You, L., \& Arnold, F. H. (1996). Directed evolution of subtilisin E inBacillus subtilis to enhance total activity in aqueous dimethylformamide. Protein Eng , 9, 77-83. doi: 10.1093/protein/9.1.77.

Zeymer, C., \& Hilvert, D. (2018). Directed evolution of protein catalysts. Annu Rev Biochem , 87, 131-157. doi: 10.1146/annurev-biochem-062917-012034.

Zhang, X. Z., Cui, Z. L., Hong, Q., \& Li, S. P. (2005). High-level expression and secretion of methyl parathion hydrolase in Bacillus subtilis WB800. Appl Environ Microbiol , 71, 4101-4103. doi: 10.1128/AEM.71.7.41014103.2005.

Zhang, X. Z., Sathitsuksanoh, N., Zhu, Z., Percival, \& Zhang, Y. H. (2011). One-step production of lactate from cellulose as the sole carbon source without any other organic nutrient by recombinant cellulolytic Bacillus subtilis . Metab Eng , 13, 364-372. doi: 10.1016/j.ymben.2011.04.003.

Zhang, X. Z., \& Zhang, Y. H. P. (2011). Simple, fast and high-efficiency transformation system for directed evolution of cellulase in Bacillus subtilis . Microb Biotechnol , 4(1), 98-105. doi: 10.1111/j.17517915.2010.00230.x.

Zhao, H., \& Arnold, F. H. (1999). Directed evolution converts subtilisin E into a functional equivalent of thermitase. Protein Eng , 12, 47-53. doi: 10.1093/protein/12.1.47.

Zhao, L. Z., Ye, B., Zhang, Q., Cheng, D., Zhou, C. Y., Cheng, S., \& Yan, X. (2019). Construction of second generation protease-deficient hosts of Bacillus subtilis for secretion of foreign proteins.Biotechnol Bioeng , 116(8), 2052-2060. doi: 10.1002/bit.26992.

TABLE 1 Primers used in this study

\begin{tabular}{lll}
\hline Primers & Sequence $\left(5^{\prime}\right.$ to $\left.3^{\prime}\right)$ & Purpose \\
\hline P1 & tgaactttatctgagaatagtcaatcttcggaaatccaggtggc & For the construction of multimer of insertion constr \\
P2 & cattttcttcctcctttcttatcataatacataattttcaaactg & \\
P3 & cagttgaaaattatgtattatgataagaaagggaggaagaaaaatg & \\
P4 & gccacctgggattccgaagattgactattctcagataaagttca & \\
P5 & cagcgcaaatgctccegctatcatcgagctccagcatccttgcagtcttcatatg & \\
P6 & catatgaagactgcaaggatgctggagctcgatgatagcgggagcatttgcgctg & \\
P7 & tttggaaagcgaggga & For the construction of variant T47C
\end{tabular}




\begin{tabular}{lll}
\hline Primers & Sequence (5' to 3') & Purpose \\
\hline P8 & ctgaacgccatcgtaaagattgacgttaacgcaaacaacaaacttatc & \\
P9 & gataagtttgttgtttgcgttaacgtcaatctttacgatggcgttcag & \\
P10 & cgttggttgtatccgtgt & \\
P11 & cgcacctgcggtgctgcagcctgagcagacatgttgctgaacgcc & For the construction of variant G81T \\
P12 & ggcgttcagcaacatgtctgctcaggctgcagcaccgcaggtgcg & \\
P13 & gcggcggacttgccgtcgatgtcgagctgggtcgtgacgctggggtcgtc & For the construction of variant T806A \\
P14 & gacgacccagcgtcacgaccagctcgacatcgacggcaagtccgccgc & \\
P15 & gccttcttgcgctccaccgcgacggacttgccgtcgatgtcgagc & For the construction of variant C821T \\
P16 & gctcgacatcgacggcaagtccgtcgcggtggagcgcaagaaggc & \\
P17 & gatgtggccgatgccggggaacgacaggtggctcgccgcgatcag & For the construction of variant C892T \\
P18 & ctgatcgcggcgagccacctgtcgttccccggcatcggccacatc & \\
P19 & gagtagttcaccggcacgaatggtagcccttgccttcggcgc & For the construction of variant G938A \\
P20 & gcgccgaaggcaagggctaccatttcgtgccggtgaactactc & \\
\hline
\end{tabular}

\section{FIGURES LEGENDS}

FIGUER 1 The scheme for insertion of epPCR products to the chromosome of B. subtilis. Firstly, fragment RF-LF-Ab $\mathrm{B}^{\mathrm{R}}$ is generated by overlap PCR and fragment GOI is amplified by error-prone PCR (epPCR). Secondly, the DNA multimer is formed by prolonged overlap extension PCR using fragments RF-LF-Ab ${ }^{\mathrm{R}}$ and GOI. Thirdly, the DNA multimers is digested to monomer (LF-Ab ${ }^{\mathrm{R}}$-GOI-RF) at the cleavage site introduced between RF and LF. Finally, the insertion construct is transformed into competent cells. $\mathrm{LF}$, left flanking region; $\mathrm{Ab}^{\mathrm{R}}$, antibiotic resistant marker; GOI, gene of interest; $\mathrm{RF}$, right flanking region; scissors, restriction endonuclease digestion.

FIGUER 2 Development of the random mutagenesis system.(a) Construction of MPH secretion strain BPC1. Promoter $P_{c r y 3 A}$ controls transcription of $m p d$ and the signal-peptide of AprE ( $\left.\mathrm{SP}_{\mathrm{AprE}}\right)$ mediates the secretion of the Methyl Parathion Hydrolase (MPH). (b) Analysis of insertion construct assembly process by agarose gel electrophoresis. The arrow indicates the position of the target DNA. (c) The effect of competent cell number on transformation efficiency of insertion construct. The amount of the insertion construct was $100 \mathrm{ng}$ and contained 1-kb flanking region. (d) The effect of flanking region size on transformation efficiency of insertion construct. The amount of the insertion construct was $100 \mathrm{ng}$ and the volume of competent cells is $400 \mu \mathrm{L}$. All data were collected from at least three biological replicates and are shown as the mean \pm SD. Bars indicated by the same letter are not significantly different $(\mathrm{P}>0.05$, evaluated by Duncan's test).

FIGUER 3 Screening of the library of MPH variants. (a) Screening of the library on LB plate containing $50 \mathrm{mg} / \mathrm{L}$ chlorpyrifos. Frist, transformants were selected on LB plates containing Zeocin. Then, the colonies were transferred to LB plates containing $50 \mathrm{mg} / \mathrm{L}$ chlorpyrifos using sterile toothpick. When chlorpyrifos are hydrolyzed by MPH, a transparent halo forms around the colony. (b) The extracellular activities of MPH in the supernatant of strain BPC1 and its mutants. All data were collected from at least three biological replicates and are shown as the mean \pm SD.

FIGUER 4 Identification of the effect of each mutation on MPH . (a) Base mutations in mpd variants. The vertical line indicates the base mutations in the variants. (b) The extracellular activities of MPH in the supernatant of strain BPC1 and six mutants with single mutation. (c) Specific activity of wild-type MPH and the five variants with single mutation. (d) SDS-PAGE analysis of extracellular MPH in the supernatant of the strain BPC1 and its mutants. (e) SDS-PAGE analysis of extracellular MPH in the supernatant of the mutants MT-C2 and MT-C3. Mutant MT-C2 harbors mutations T47C and T806A, and mutant MT-C3 contains mutations of T47C, T806A and G938A. The strains were grown on $2 \times \mathrm{SR}$ medium and incubated at $37^{\circ} \mathrm{C}$ with shaking at $200 \mathrm{rpm}$ for $36 \mathrm{~h}$. Equal amounts $(20 \mu \mathrm{l})$ of culture supernatant were loaded into each lane. (f) The extracellular activities of MPH in the supernatant of strain BPC1, MT-C2 and 
MT-C3. M, protein markers. The arrow indicates the position of the target band. All data were collected from at least three biological replicates and are shown as the mean \pm SD. Bars indicated by the same letter are not significantly different $(\mathrm{P}>0.05$, evaluated by Duncan's test).

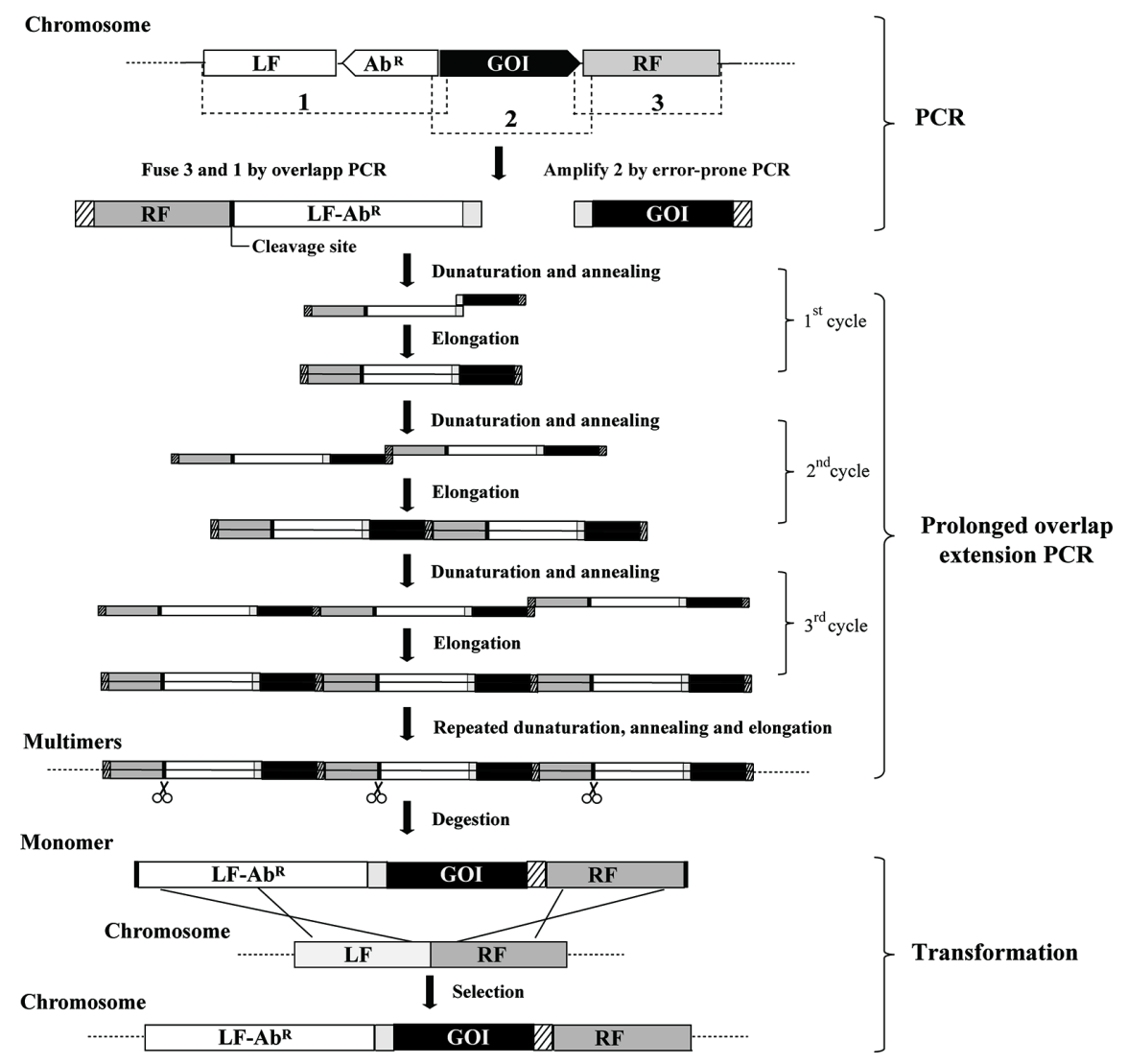


(a)

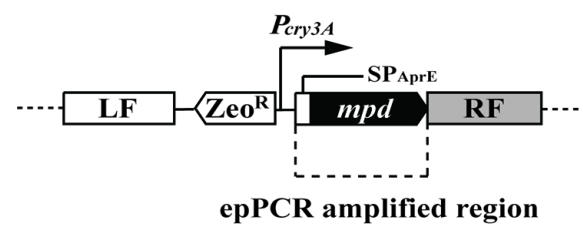

(c)

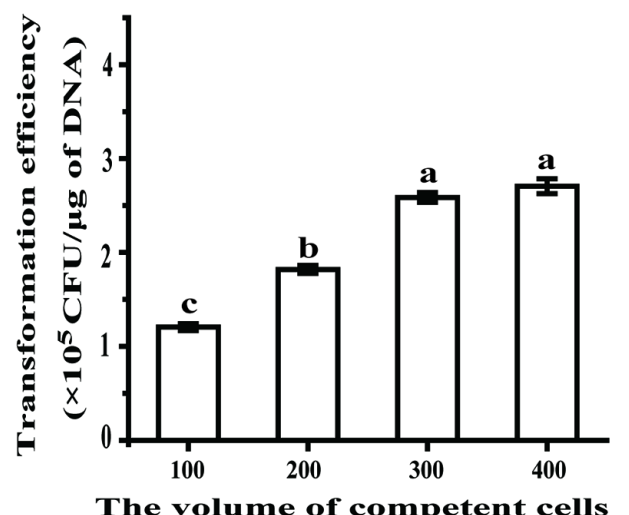
(ML)

(a)

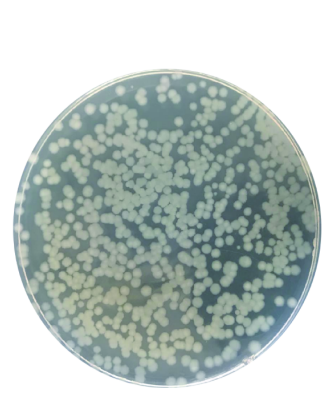

(b)

(d)
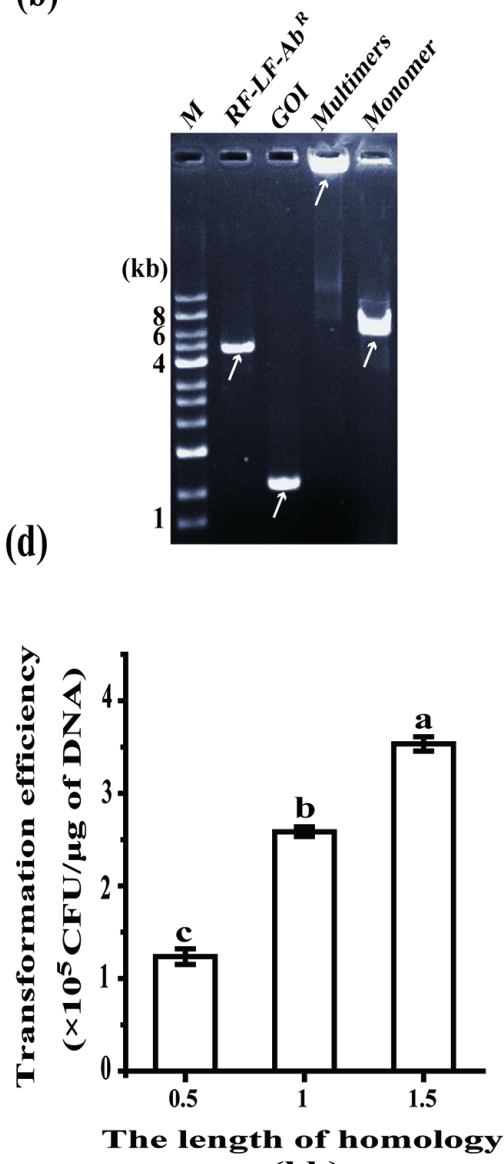

(kb)

(b)

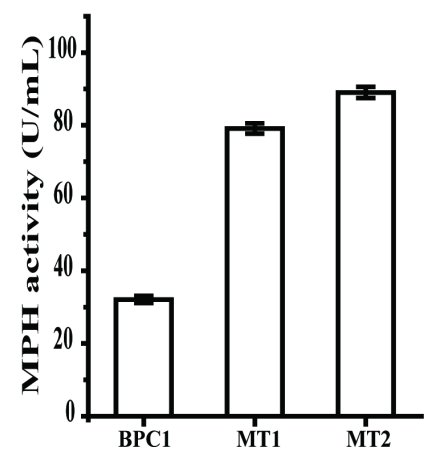


(a)

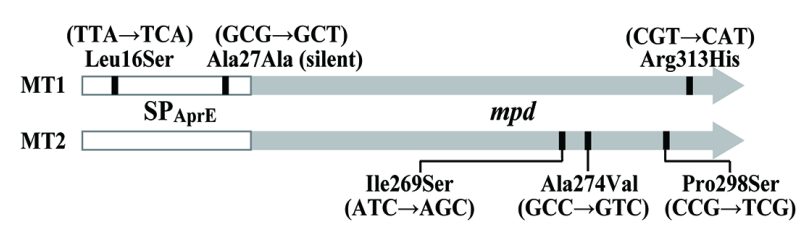

(b)

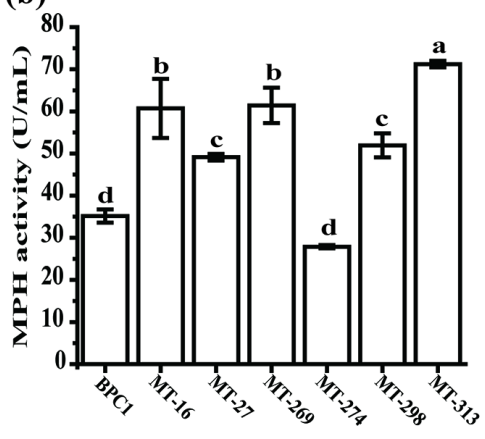

(d)

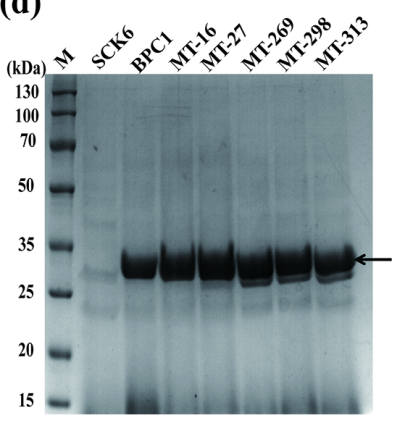

(c)

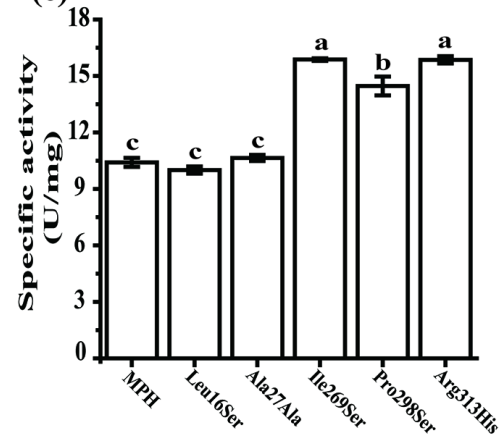

(f)

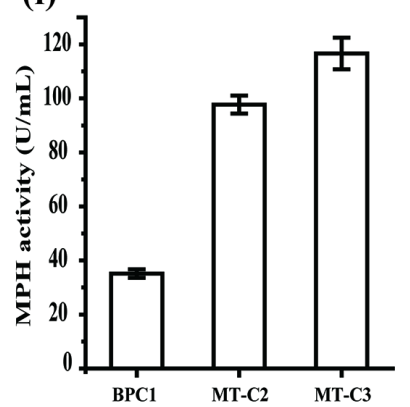

